\title{
Quadrotor Accelerometer and Gyroscope Sensor Fault Diagnosis Using Nonlinear Adaptive Estimation Methods
}

\author{
Remus Avram ${ }^{1}$, Xiaodong Zhang ${ }^{2}$, and Jonathan Muse ${ }^{3}$ \\ ${ }^{1,2}$ Wright State University, Dayton OH, 45404, USA \\ avram.3@wright.edu \\ xiaodong.zhang@wright.edu \\ ${ }^{3}$ Air Force Research Laboratory, Dayton OH, 45433, USA \\ jonathan.muse.2@us.af.mil
}

\begin{abstract}
This paper presents the design, analysis, and real-time experimental evaluation results of a nonlinear sensor fault diagnosis scheme for quadrotor unmanned air vehicles (UAV). The objective is to detect, isolate, and estimate sensor bias faults in accelerometer and gyroscope measurements. Based on the quadrotor dynamics and sensor models under consideration, the effects of sensor faults are represented as virtual actuator faults in the quadrotor state equation. Two nonlinear diagnostic estimators are designed to provide structured residuals for fault detection and isolation. Additionally, after the fault is detected and isolated, a nonlinear adaptive estimation scheme is employed for estimating the unknown fault magnitude. The proposed fault diagnosis scheme is capable of handling simultaneous faults in the accelerometer and gyroscope measurements. The effectiveness of the fault diagnosis method is demonstrated using an indoor real-time quadrotor UAV test environment.
\end{abstract}

\section{INTRODUCTION}

Unmanned Aerial Vehicles (UAVs) have attracted significant attentions in the recent decade due to their potentials in various military and civilian applications, including security patrol, search and rescue in hazardous environment, surveillance and classification, attack and rendezvous (Shima \& Rasmussen, 2008). In addition, compared with manned systems, the reductions in operations and support costs offer the advantage for life cycle cost savings (US Dept. of Defense, 2012). The potential capabilities offered by unmanned vehicles have

Remus Avram et al. This is an open-access article distributed under the terms of the Creative Commons Attribution 3.0 United States License, which permits unrestricted use, distribution, and reproduction in any medium, provided the original author and source are credited.

Distribution Statement A, Approved for Public Release; Distribution Unlimited. Cleared by 88 ABW/PA, 22 JUL 2015 (88ABW-2015-3703) been well recognized and continue to expand. In manned systems, the human operator functions as the central integrator of the on-board systems to achieve their operational capabilities. Due to the requirement of autonomous operations without a human operator, autonomous control of UAVs is much more challenging. In order to enhance the reliability, survivability, and autonomy of UAVs, advanced intelligent control and health management technologies are required (Y. Zhang et al., 2013; Vachtsevanos, Tang, Drozeski, \& Gutierrez, 2005).

Quadrotors are often equipped with low-cost and lightweight micro-electro-mechanical systems (MEMS) inertial measurement units (IMU) including 3-axis gyroscope, accelerometer and magnetometer. These sensors serve an essential role in most quadrotor control schemes. However, IMU measurements are susceptible to sensor bias faults as a result of temperature variation, vibration, component damage, etc.

Despite a steady growth of quadrotor applications, on-board sensor fault diagnosis has received limited consideration. Most fault diagnosis methods with application to quadrotors deal exclusively with actuator faults (Y. Zhang et al., 2013). Additionally, small UAV sensor fault diagnosis methods found in literature are often designed based on linearized system models around certain equilibrium points using linear observers or Kalman filters. However, quadrotor dynamics are inherently nonlinear, especially during dynamic transient operations. Therefore, there is significant research interest in fault diagnosis methods that are directly based on the intrinsic nonlinearities of quadrotor dynamics. The extended Kalman filter (EKF) is a commonly used method to deal with nonlinear systems. However, a stability analysis for EKF applied to parameter estimation of nonlinear systems is very difficult. Furthermore, there have been very limited real-time experimental results on quadrotor sensor fault diagnosis (Y. Zhang et al., 2013; Freddi, Longhi, \& Monteriú, 2009).

In this paper, a nonlinear method for detecting, isolating, and 
estimating sensor bias faults in accelerometer and gyroscope measurements of quadrotor UAVs is presented. The stability and convergence properties of the adaptive fault parameter scheme is also analyzed. Based on the fact that the accelerometer and gyroscope measure the specific forces/angular rates acting on the UAV body, the quadrotor dynamics are represented in terms of IMU measurements. Specifically, the measurements provided by the accelerometer are expressed as inputs in the quadrotor translational dynamics, and the measurements provided by the gyroscope are expressed as inputs in the quadrotor rotational dynamics. Thus, the measurements provided by the IMU sensors are represented as virtual actuators in the quadrotor dynamics, and the effects of IMU sensor biases are treated as virtual actuator faults. Two robust diagnostic estimators are designed to provide structured fault detection and isolation (FDI) residuals enabling the detection and isolation of simultaneous gyroscope and accelerometer sensor faults. In addition, by utilizing nonlinear adaptive estimation techniques (X. Zhang, Polycarpou, \& Parsini, 2001; Bastin \& Gevers, 1988), adaptive estimators are employed to provide an estimate of the unknown sensor bias. The stability and parameter convergence properties of the adaptive estimation scheme are analyzed in the presence of sensor measurement noise. The sensor fault diagnosis method is implemented using a real-time indoor quadrotor test environment. Real-time experimental results are shown to illustrate the effectiveness of the diagnostic method.

The remainder of the paper is organized as follows. Section 2 formulates the problem of sensor FDI for quadrotor UAVs. The proposed fault detection and isolation method is presented in Section 3. Section 4 describes the adaptive algorithms for estimating sensor bias magnitude. Section 5 describes the real-time quadrotor test environment and some experimental results to demonstrate the effectiveness of the method. Finally, conclusions and directions of future research are given in Section 6.

\section{Problem formulation}

Several researchers have investigated quadrotor modeling, aiming for high fidelity models by including drag force, Coriolis effects, blade flapping effects, rotor dynamics, etc. (Castillo, Lozano, \& Dzul, 2005; Pounds, Mahony, \& Gresham, 2004; Bangura \& Mahony, 2012). Accurate modeling plays an important role in quadrotor control, especially in the case of aggressive maneuvers, tight group formations, etc. However, when the quadrotor is in a non-aggressive flight, these effects become very small in comparison to gravitational pull and thrust generated by the rotors. As in (Leishman, McDonald, Beard, \& McLain, 2014; Martin \& Salaün, 2010), the dynamic model used in this paper considers the gravity, thrust generated by the rotors, and drag forces acting on the quadrotor body. The quadrotor nominal system dynamics derived from the Newton-Euler equations of motion are given by:

$$
\begin{aligned}
& \dot{p}_{E}=v_{E} \\
& \dot{v}_{E}=\frac{1}{m} R_{E B}(\eta)\left(\left[\begin{array}{c}
0 \\
0 \\
-U
\end{array}\right]-f_{\mathbf{D}}\right)+\left[\begin{array}{l}
0 \\
0 \\
g
\end{array}\right] \\
& \dot{\eta}=\left[\begin{array}{ccc}
1 & \sin \phi \tan \theta & \cos \phi \tan \theta \\
0 & \cos \phi & -\sin \phi \\
0 & \sin \phi \sec \theta & \cos \phi \sec \theta
\end{array}\right] \omega \\
& {\left[\begin{array}{c}
\dot{p} \\
\dot{q} \\
\dot{r}
\end{array}\right]=\left[\begin{array}{l}
\frac{J_{y}-J_{z}}{J_{x}} q r \\
\frac{J_{z}-J_{x}}{J_{y}} p r \\
\frac{J_{x}-J_{y}}{J_{z}} p q
\end{array}\right]+\left[\begin{array}{c}
\frac{1}{J_{x}} \tau_{\phi} \\
\frac{1}{J_{y}} \tau_{\theta} \\
\frac{1}{J_{z}} \tau_{\psi}
\end{array}\right],}
\end{aligned}
$$

where $p_{E} \in \mathbb{R}^{3}$ is the inertial position, $v_{E} \in \mathbb{R}^{3}$ is the velocity expressed in the Earth frame, $\eta=[\phi, \theta, \psi]^{T}$ are the roll, pitch and yaw Euler angles, respectively, and $\omega=[p, q, r]^{T}$ represents the angular rates, $m$ is the mass of the quadrotor, and $g$ is the gravitational acceleration. The terms $J_{x}, J_{y}$ and $J_{z}$ represent the quadrotor inertias about the body $\mathrm{x}-, \mathrm{y}-$ and $\mathrm{z}$-axis, respectively. Note that the quadrotor is assumed to be symmetric about the $x z$ and $y z$ body planes (i.e. the product of inertias is zero). $U$ represents the total thrust generated by the rotors, $\tau_{\phi}, \tau_{\theta}, \tau_{\psi}$ are the torques acting on the quadrotor around the body $\mathrm{x}-, \mathrm{y}$ - and $\mathrm{z}$-axis, respectively. Finally, the term $f_{\mathbf{D}}$ represents the translational drag force acting on the vehicle frame.

The system model described by Eq. (1) - Eq. (4) is expressed with the velocity relative to the inertial frame. The inertial coordinate system is assumed to have the positive $\mathrm{X}$-axis pointing North, the positive $\mathrm{y}$-axis pointing East, and positive $\mathrm{z}$ axis pointing down towards the Earth's center. The transformation from the body frame to inertial frame is given by the rotation matrix $R_{E B}$ and is defined based on a 3-2-1 rotation sequence as follows:

$$
R_{E B}(\eta)=\left[\begin{array}{ccc}
c \theta c \psi & s \phi s \theta c \psi-c \phi s \psi & c \phi s \theta c \psi+s \phi s \psi \\
c \theta s \psi & s \phi s \theta s \psi+c \phi c \psi & c \phi s \theta s \psi-s \phi c \psi \\
-s \theta & s \phi c \theta & c \phi c \theta
\end{array}\right]
$$

where $s \cdot$ and $c$ are shorthand notations for the $\sin (\cdot)$ and $\cos (\cdot)$ functions, respectively. As in (Leishman et al., 2014), by assuming that the nonlinear Coriolis terms are small enough to be negligible, the quadrotor velocity dynamics relative to the body frame are expressed as:

$$
\left[\begin{array}{c}
\dot{u}_{x} \\
\dot{u}_{y} \\
\dot{u}_{z}
\end{array}\right]=\frac{1}{m}\left(\left[\begin{array}{c}
0 \\
0 \\
-T
\end{array}\right]-f_{\mathbf{D}}\right)+\left[\begin{array}{c}
-g \sin \theta \\
g \sin \phi \cos \theta \\
g \cos \phi \cos \theta
\end{array}\right],
$$

where $v_{B} \triangleq\left[u_{x}, u_{y}, u_{z}\right]^{T}$, represents the velocities along the body $x-, y-$ and $z-$ directions. The relationship be- 
tween the inertial velocity and body velocity is given by:

$$
v_{E}=R_{E B} v_{B} .
$$

As in (Freddi et al., 2009; Ireland \& Anderson, 2012; Lantos \& Marton, 2011), it is assumed that Euler angles measurements are available. For instance, these measurements can be generated by a camera-based motion capture system, a technology commonly employed for in-door UAV flight (Leishman et al., 2014; Ireland \& Anderson, 2012; Guenard, Hamel, \& Mahony, 2008; Dydek, Annaswamy, \& Lavretsky, 2013).

MEMS sensors, such as accelerometers and gyroscopes, measure forces and moments acting in the body frame. By considering IMU measurements susceptibility to bias faults, the accelerometer and gyroscope sensor measurements are given by:

$$
\begin{aligned}
& y_{a}=a+\beta_{a}\left(t-t_{a}\right) b_{a}+n_{a} \\
& y_{\omega}=\omega+\beta_{\omega}\left(t-t_{\omega}\right) b_{\omega}+n_{\omega}
\end{aligned}
$$

where $y_{a} \in \mathbb{R}^{3}$ and $y_{\omega} \in \mathbb{R}^{3}$ are the accelerometer and gyroscope measurements, respectively, $b_{a} \in \mathbb{R}^{3}$ and $b_{\omega} \in \mathbb{R}^{3}$ represent sensor bias faults in accelerometer and gyroscope measurements, respectively, the terms $n_{a}$ and $n_{\omega}$ represent the measurement noise, and:

$$
a=\frac{1}{m}\left(\left[\begin{array}{c}
0 \\
0 \\
-U
\end{array}\right]-f_{\mathbf{D}}\right)
$$

represents the nominal acceleration measurement without bias and noise. Additionally, $\beta_{a}(\cdot)$ and $\beta_{\omega}(\cdot)$ are fault time profile functions with unknown fault occurrence times $t_{a}$ and $t_{\omega}$, respectively. In this paper, they are modeled as step functions given by:

$$
\begin{aligned}
& \beta_{a}\left(t-t_{a}\right)= \begin{cases}0, & \text { when } t<t_{a} \\
1, & \text { when } t \geq t_{a}\end{cases} \\
& \beta_{\omega}\left(t-t_{\omega}\right)= \begin{cases}0, & \text { when } t<t_{\omega} \\
1, & \text { when } t \geq t_{\omega}\end{cases}
\end{aligned}
$$

It is assumed that the position measurements in the Earth frame are available. Hence, the system model is augmented by the following output equation:

$$
y_{p}=p_{E}+d_{p}
$$

where $d_{p}$ represents zero mean position measurement noise.

Assumption 1. The sensor biases $b_{a}$ and $b_{\omega}$ in Eq. (6) and Eq. (7) are assumed to be constant and bounded.

Assumption 2. The sensor measurement noises, denoted by $n_{a}, n_{\omega}$ and $d_{p}$ in Eq. (6), Eq. (7), and Eq. (9), respectively, are assumed to be bounded zero mean signals. That is:

$$
\mathbb{E}\left(n_{a}\right)=0, \quad \mathbb{E}\left(n_{\omega}\right)=0, \quad \mathbb{E}\left(d_{p}\right)=0,
$$

where $\mathbb{E}$ represents the expectation operator.

Remark 1. It is worth noting that, in practical applications, after the occurrence of an IMU sensor bias, its magnitude may be time-varying and grow slowly over time. However, the change in the bias is often small over a short time duration. Therefore, the bias may be assumed to be constant on the short time duration under consideration.

The objective of this research focuses on the design, analysis, and experimental demonstration of a robust fault detection, isolation, and estimation scheme for sensor bias faults in accelerometer and gyroscope measurements described by Eq. (6) and Eq. (7).

\section{FAult Detection ANd Isolation Method}

This section presents the proposed method for detecting and isolating sensor faults in accelerometer and gyroscope measurements. Substituting the sensor model given by Eq. (6) - Eq. (7) into the systems dynamics Eq. (1) - Eq. (4), we obtain:

$$
\begin{aligned}
\dot{p}_{E} & =v_{E} \\
\dot{v}_{E} & =R_{E B}(\eta) y_{a}+\left[\begin{array}{l}
0 \\
0 \\
g
\end{array}\right]-R_{E B}(\eta) \beta_{a} b_{a}-R_{E B} n_{a} \\
\dot{\eta} & =R_{\eta}(\phi, \theta) y_{\omega}-R_{\eta}(\phi, \theta) \beta_{\omega} b_{\omega}-R_{\eta}(\phi, \theta) n_{\omega} \\
\dot{\omega} & =\left[\begin{array}{l}
\frac{J_{y}-J_{z}}{J_{x}}\left(y_{q}-\beta_{\omega} b_{q}-d_{q}\right)\left(y_{r}-\beta_{\omega} b_{r}-d_{\eta}\right) \\
\frac{J_{z}-J_{x}}{J_{y}}\left(y_{r}-\beta_{\omega} b_{r}-d_{r}\right)\left(y_{p}-\beta_{\omega} b_{p}-d_{\eta}\right) \\
\frac{J_{x}-J_{y}}{J_{z}}\left(y_{p}-\beta_{\omega} b_{p}-d_{p}\right)\left(y_{q}-\beta_{\omega} b_{q}-d_{q}\right)
\end{array}\right]+\left[\begin{array}{c}
\frac{1}{J_{x}} \tau_{\phi} \\
\frac{1}{J_{y}} \tau_{\theta} \\
\frac{1}{J_{z}} \tau_{\psi}
\end{array}\right]
\end{aligned}
$$

where $R_{\eta}(\phi, \theta)$ is the rotation matrix relating angular rates to Euler angle rates and is given by:

$$
R_{\eta}(\phi, \theta)=\left[\begin{array}{ccc}
1 & \sin \phi \tan \theta & \cos \phi \tan \theta \\
0 & \cos \phi & -\sin \phi \\
0 & \sin \phi \sec \theta & \cos \phi \sec \theta
\end{array}\right]
$$

As can be seen from Eq. (10) - Eq. (13), a bias in accelerometer measurements affects only the position and velocity states. On the other hand, gyroscope measurements affect only Euler angles and angular rate states. Based on this observation, the effect of a bias in either sensor measurements can be treated as a virtual actuator fault. In addition, due to the decoupling of the two sensor faults in the quadrotor state equations, it follows naturally to also divide the fault diagnosis tasks of these two types of sensor faults. The proposed fault diagnosis architecture is shown in Figure 1. As can be seen, under normal operating conditions, two FDI estimators monitor the system for detecting and isolating fault occurrences in accelerometer and gyroscope measurements. Once a fault is detected and isolated, the corresponding nonlinear adaptive estimator 


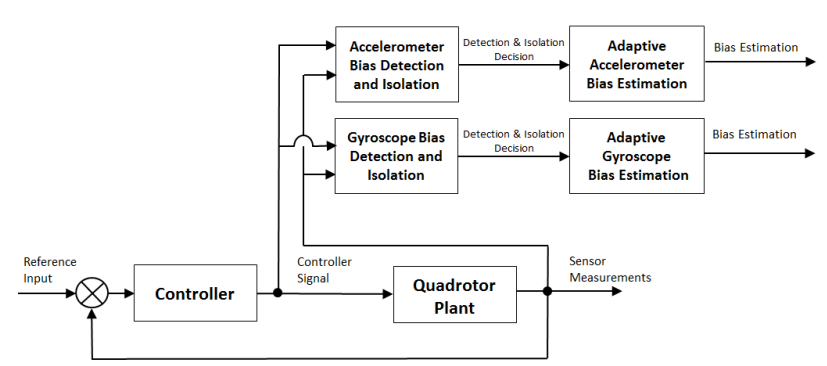

Figure 1. Sensor fault diagnosis architecture.

is activated for sensor bias estimation.

\subsection{Gyroscope Fault Diagnostic Estimator}

Based on Eq. (12), the fault diagnostic estimator for the gyroscope bias can be designed as follows:

$$
\dot{\hat{\eta}}=-\Lambda_{1}(\hat{\eta}-\eta)+R_{\eta}(\phi, \theta) y_{\omega},
$$

where $\hat{\eta} \in \mathbb{R}^{3}$ are the Euler angle estimates, and $\Lambda_{1} \in \mathbb{R}^{3 \times 3}$ is a positive-definite diagonal design matrix. Let the Euler angle estimation error be defined as:

$$
\tilde{\eta} \triangleq \eta-\hat{\eta} .
$$

Based on Eq. (12), Eq. (14) and Eq. (15), the dynamics of the attitude angle estimation error are given by:

$$
\dot{\tilde{\eta}}=\dot{\eta}-\dot{\hat{\eta}}=-\Lambda_{1} \tilde{\eta}-R_{\eta}(\phi, \theta) \beta_{\omega} b_{\omega}-R_{\eta}(\phi, \theta) n_{\omega} .
$$

In the absence of a gyroscope fault (i.e. for $t<t_{\omega}$ ), the attitude angle estimation error is given by:

$$
\begin{aligned}
\tilde{\eta}(t) & =e^{-\Lambda_{1}\left(t-t_{\omega}\right)} \tilde{\eta}(0)-\int_{0}^{t} e^{-\Lambda_{1}(t-\tau)} R_{\eta}(\phi, \theta) n_{\omega} d \tau \\
& =r_{\omega}(t)+e_{\omega}(t)
\end{aligned}
$$

where $r_{\omega}(t) \triangleq e^{-\Lambda_{1}\left(t-t_{\omega}\right)} \tilde{\eta}(0)$ converges exponentially to zero, and

$$
e_{\omega}(t) \triangleq-\int_{0}^{t} e^{-\Lambda_{1}(t-\tau)} R_{\eta}(\phi, \theta) n_{\omega} d \tau
$$

represents an additive zero mean noise term generated by filtering the measurement noise $n_{\omega}$ through the following stable linear filter:

$$
\dot{e}_{\omega}=-\Lambda_{1} e_{\omega}-R_{\eta}(\phi, \theta) n_{\omega} .
$$

Therefore, $\mathbb{E}(\tilde{\eta})$ converges exponentially to zero in the absence of faults. In addition, based on Eq. (16), it can be seen that the residual $\tilde{\eta}$ is only sensitive to gyroscope sensor bias $b_{\omega}$. Thus, if $\mathbb{E}(\tilde{\eta})$ is significantly different from zero, it can be concluded that a fault in the gyroscope measurements has occurred.

\subsection{Accelerometer Fault Diagnostic Estimator}

The translational dynamics of the quadrotor described by Eq. (10) and Eq. (11) can be rewritten as follows:

$$
\begin{aligned}
& \dot{x}=A x+f\left(\eta, y_{a}\right)+G_{a}(\eta) \beta_{a} b_{a}+D_{a}(\eta, t) \\
& y=C x+d_{p}
\end{aligned}
$$

where $x=\left[p_{E}^{T}, v_{E}^{T}\right]^{T}, y=p_{E}$,

$$
A=\left[\begin{array}{cc}
0_{3 \times 3} & I_{3} \\
0_{3 \times 3} & 0_{3 \times 3}
\end{array}\right], \quad G_{a}(\eta)=\left[\begin{array}{c}
0_{3 \times 3} \\
-R_{E B}
\end{array}\right],
$$

$f\left(\eta, y_{a}\right)=\left[\begin{array}{c}0_{3 \times 1} \\ R_{E B} y_{a}+\left[\begin{array}{l}0 \\ 0 \\ g\end{array}\right]\end{array}\right], \quad D_{a}(\eta, t)=\left[\begin{array}{c}0_{3 \times 1} \\ -R_{E B} n_{a}\end{array}\right]$,

and $C=\left[I_{3}, 0_{3 \times 3}\right]$, where $I_{3}$ is a $3 \times 3$ identity matrix, $0_{3 \times 3}$ is a $3 \times 3$ matrix with all entries zeros, and $0_{3 \times 1}$ is a $3 \times 1$ zero vector. Based on Eq. (18), the following fault diagnostic observer is chosen:

$$
\begin{aligned}
& \dot{\hat{x}}=A \hat{x}+f\left(\eta, y_{a}\right)+L_{1}(y-\hat{y}) \\
& \hat{y}=C \hat{x}
\end{aligned}
$$

where $\hat{x} \in \mathbb{R}^{6}$ represents the inertial position and velocity estimation, $\hat{y} \in \mathbb{R}^{3}$ are the estimated position outputs, $L_{1}$ is a design matrix chosen such that the matrix $\bar{A}_{1} \triangleq\left(A-L_{1} C\right)$ is asymptotically stable. Note that $(A, C)$ is an observable pair.

Let us define the position estimation error as:

$$
\tilde{y} \triangleq y-\hat{y},
$$

and the state estimation error as:

$$
\tilde{x} \triangleq x-\hat{x} .
$$

By using equations Eq. (18) - Eq. (19), the estimation error dynamics are given by:

$$
\begin{aligned}
\dot{\tilde{x}} & =\bar{A}_{1} \tilde{x}+G_{a}(\eta) \beta_{a} b_{a}+D_{a}(\eta, t)-L_{1} d_{p} \\
\tilde{y} & =C \tilde{x}+d_{p} .
\end{aligned}
$$

In the absence of accelerometer bias (i.e. for $t<t_{a}$ ), the position estimation error is given by:

$$
\begin{aligned}
\tilde{y} & =C e^{\bar{A}_{1}\left(t-t_{a}\right)} \tilde{x}(0)+C \int_{0}^{t} e^{\bar{A}_{1}(t-\tau)}\left(D_{a}(\eta, t)-L_{1} d_{p}\right) d \tau+d_{p} \\
& =r_{a}(t)+e_{a}(t)+d_{p}
\end{aligned}
$$

where $r_{a}(t) \triangleq C e^{\bar{A}_{1}\left(t-t_{a}\right)} \tilde{x}(0)$ converges exponentially to zero, and

$$
e_{a}(t) \triangleq C \int_{0}^{t} e^{\bar{A}_{1}(t-\tau)}\left(D_{a}(\eta, t)-L_{1} d_{p}\right) d \tau
$$


represents an additive zero mean noise term generated by filtering $n_{a}$ and $d_{p}$ through the following stable linear filter:

$$
\dot{e}_{a}=\bar{A}_{1} e_{a}+\left(D_{a}(\eta, t)-L_{1} d_{p}\right) .
$$

Clearly, $\mathbb{E}(\tilde{y})$ reaches zero exponentially in the absence of the accelerometer bias $b_{a}$. Furthermore, it can be seen from Eq. (22), the residual $\tilde{y}$ is only sensitive to the bias $b_{a}$. Therefore, if any component of $\mathbb{E}(\tilde{y})$ deviates significantly from zero, it can be concluded that a fault in the accelerometer sensor measurement has occurred.

\subsection{Fault Detection and Isolation Decision Scheme}

As described in Sections 3.1 and 3.2, the two fault diagnostic estimators are designed such that each of them is only sensitive to one type of sensor faults. Based on this observation, the residuals $\tilde{\eta}$ and $\tilde{y}$ generated by Eq. (17) and Eq. (23) can also be used as structured residuals for fault isolation. More specifically, the following fault detection and isolation decision scheme is formulated:

- In the absence of any faults, all components of $\mathbb{E}(\tilde{\eta})$ and $\mathbb{E}(\tilde{y})$ should be close to zero.

- If all components of $\mathbb{E}(\tilde{\eta})$ remain around zero, and at least one component of $\mathbb{E}(\tilde{y})$ is significantly different from zero, then it can be concluded that an accelerometer fault has occurred.

- If all components of $\mathbb{E}(\tilde{y})$ remain around zero, and at least one component of $\mathbb{E}(\tilde{\eta})$ is significantly different from zero, then it can be concluded that a gyroscope fault has occurred.

- If at least one component of $\mathbb{E}(\tilde{\eta})$ and at least one component of $\mathbb{E}(\tilde{y})$ are both significantly different from zero, then it can be concluded that both a gyroscope and an accelerometer sensor measurement fault has occurred.

The above FDI decision scheme is summarized in Table 1, where " 0 " represents residuals with zero mean, and " 1 " represents significantly non-zero residuals.

Table 1. Fault Isolation Decision Truth Table.

\begin{tabular}{|l|c|c|c|c|}
\hline & No Fault & Gyro Bias & Accel Bias & $\begin{array}{c}\text { Accel \& Gyro } \\
\text { Bias }\end{array}$ \\
\hline $\mathbb{E}(\tilde{\eta})$ & 0 & 1 & 0 & 1 \\
\hline $\mathbb{E}(\tilde{y})$ & 0 & 0 & 1 & 1 \\
\hline
\end{tabular}

\section{Fault Estimation}

As shown in Figure 1, once a sensor fault is detected and isolated, the corresponding nonlinear adaptive estimator is activated for estimating the unknown fault magnitude in the accelerometer and/or gyroscope measurements. In this section, the design of nonlinear adaptive estimators for sensor bias estimation is described.

\subsection{Accelerometer Fault Estimation}

Based on Eq. (18), the adaptive observer for estimating the accelerometer bias magnitude is chosen as:

$$
\begin{aligned}
\dot{\hat{x}} & =A \hat{x}+f\left(\eta, y_{a}\right)+L_{2}(y-\hat{y})+G_{a}(\eta) \hat{b}_{a}+\Omega \dot{\hat{b}}_{a} \\
\dot{\Omega} & =\left(A-L_{2} C\right) \Omega+G_{a}(\eta) \\
\hat{y} & =C \hat{x},
\end{aligned}
$$

where $\hat{x}$ is the estimated position and velocity vector, $\hat{y}$ is the estimated position output, $\hat{b}_{a}$ is the estimated sensor bias, and $L_{2}$ is the observer gain matrix. The adaptation in the above adaptive estimator arises due to the unknown bias $b_{a}$. The adaptive law for updating $\hat{b}_{a}$ is derived using Lyapunov synthesis approach (Bastin \& Gevers, 1988; X. Zhang, 2011) and is given by:

$$
\dot{\hat{b}}_{a}=\Gamma \Omega^{T} C^{T} \tilde{y},
$$

where $\Gamma>0$ is a symmetric and positive-definite learning rate matrix, and $\tilde{y}_{a} \triangleq y_{a}-\hat{y}_{a}$ is the output estimation error. Let us also define the state estimation error as $\tilde{x} \triangleq x-\hat{x}$, and the parameter estimation error as $\tilde{b}_{a} \triangleq \hat{b}_{a}-b_{a}$. The stability and performance properties of the above adaptive scheme are described below.

Theorem 1. Suppose that an accelerometer sensor bias fault occurs at $t_{a} \geq 0$ and is detected at some time $T_{a}>t_{a}$. Then, if there exists constants $\alpha_{1} \geq \alpha_{0}>0$ and $T_{0}>0$, such that $\forall t>T_{a}$

$$
\alpha_{1} I \geq \frac{1}{T_{0}} \int_{t}^{t+T_{0}} \Omega^{T} C^{T} C \Omega d \tau \geq \alpha_{0} I,
$$

the adaptive fault estimation scheme described by Eq. (24) Eq. (27) guarantees that:

1. all signals in the adaptive estimator remain bounded;

2. $\mathbb{E}(\tilde{x})$ and $\mathbb{E}\left(\tilde{b}_{a}\right)$ converge exponentially to zero.

Proof. Based on Eq. (18) and Eq. (24), the dynamics governing the state estimation error dynamics are given by

$$
\dot{\tilde{x}}=\bar{A}_{2} \tilde{x}-G_{a}(\eta) \tilde{b}_{a}-\Omega \dot{\hat{b}}_{a}+D_{a}(\eta, t)-L_{2} d_{p},
$$

where $\bar{A}_{2} \triangleq A-L_{2} C$ and $\tilde{b}_{a} \triangleq \hat{b}_{a}-b_{a}$ is the parameter estimation error. By substituting $G_{a}(\eta)=\dot{\Omega}-\left(A-L_{2} C\right) \Omega$ (see Eq. (25)) into Eq. (29), we have

$$
\begin{aligned}
\dot{\tilde{x}} & =\bar{A}_{2} \tilde{x}-\left(\dot{\Omega}-\bar{A}_{2} \Omega\right) \tilde{b}_{a}-\Omega \dot{\hat{b}}_{a}+D_{a}(\eta, t)-L_{2} d_{p} \\
& =\bar{A}_{2}\left(\tilde{x}+\Omega \tilde{b}_{a}\right)-\dot{\Omega} \tilde{b}_{a}-\Omega \dot{\tilde{b}}_{a}+D_{a}(\eta, t)-L_{2} d_{p} .
\end{aligned}
$$

Note that, based on Asumption 1, we have $\dot{\tilde{b}}_{a}=\dot{\hat{b}}_{a}$. By defin- 
ing $\bar{x} \triangleq \tilde{x}+\Omega \tilde{b}_{a}$, the above equation can be rewritten as:

$$
\dot{\bar{x}}=\bar{A}_{2} \bar{x}+D(\eta, t)-L_{2} d_{p},
$$

where $\bar{A}_{2}$ is asymptotically stable by design (note that the pair $(A, C)$ is observable by design). By noting that the terms $D(\eta, t)$ and $L_{2} d_{p}$ in Eq. (31) are bounded (see Assumption 2 ), it follows that $\bar{x}$ is also bounded. By using the adaptive parameter estimation algorithm Eq. (27) and $\tilde{y}=C(\bar{x}-$ $\Omega \tilde{b})+d_{p}$, we obtain

$$
\begin{aligned}
\dot{\tilde{b}}_{a} & =\Gamma \Omega^{T} C^{T} \tilde{y} \\
& =-\Gamma \Omega^{T} C^{T} C \Omega \tilde{b}_{a}+\Gamma \Omega^{T} C^{T} C \bar{x}+\Gamma \Omega^{T} C^{T} d_{p} .
\end{aligned}
$$

Note the condition given by Eq. (28) provides the required persistent excitation condition for parameter convergence (Ioannou \& Sun, 1996). Thus, using this property in conjunction with Theorem 2.2 from (Anderson et al., 1986), it follows that the homogeneous part of Eq. (32) is exponentially stable. In addition, as can be seen from Eq. (25), $\Omega$ is also bounded, which along with Assumption 2 implies that all signals in Eq. (32) are bounded. Because $\bar{x}, \Omega$ and $\tilde{b}_{a}$ are bounded, it then follows that $\tilde{x}$ is also bounded. This concludes the proof of the first part of the theorem.

By taking the expectation of Eq. (31) and by using $\mathbb{E}\left(n_{a}\right)=0$ and $\mathbb{E}\left(d_{p}\right)=0$, we have:

$$
\frac{d}{d t}(\mathbb{E}(\bar{x}))=\bar{A}_{2} \mathbb{E}(\bar{x})+\mathbb{E}\left(D_{a}(\eta, t)-L_{2} d_{p}\right)=\bar{A}_{2} \mathbb{E}(\bar{x}) .
$$

Clearly, $\mathbb{E}(\bar{x})$ converges to zero exponentially because of the stability properties of $\bar{A}_{2}$. By taking the expectation of Eq. (32), we have

$$
\begin{aligned}
\frac{d}{d t}\left(\mathbb{E}\left(\tilde{b}_{a}\right)\right)= & -\Gamma \Omega^{T} C^{T} C \Omega \mathbb{E}\left(\tilde{b}_{a}\right)+\Gamma \Omega^{T} C^{T} C \mathbb{E}(\bar{x}) \\
& +\Gamma \Omega^{T} C^{T} \mathbb{E}\left(d_{p}\right) \\
= & -\Gamma \Omega^{T} C^{T} C \Omega \mathbb{E}\left(\tilde{b}_{a}\right) .
\end{aligned}
$$

Thus, based on the persistent of excitation condition Eq. (28), it follows from Eq. (34) that $\mathbb{E}\left(\tilde{b}_{a}\right)$ converges to zero exponentially. Finally, by applying the above results to the definition of $\bar{x}$, the second part of the theorem can be concluded.

\subsection{Gyroscope Fault Estimation}

Based on Eq. (12), after the occurrence of a gyroscope bias fault is detected and isolated, the following adaptive estimator is designed in order to estimate the unknown sensor bias:

$$
\begin{aligned}
\dot{\hat{\eta}} & =-\Lambda_{2}(\hat{\eta}-\eta)+R_{\eta}(\phi, \theta) y_{\omega}-R_{\eta}(\phi, \theta) \hat{b}_{\omega} \\
\dot{\hat{b}}_{\omega} & =\Gamma R_{\eta}(\phi, \theta)^{T}(\hat{\eta}-\eta)
\end{aligned}
$$

where $\hat{\eta}$ is the Euler angle estimate, $\hat{b}_{\omega}$ represents the estimation of the sensor bias, $\Lambda$ and $\Gamma$ are positive definite design matrices. The adaptive law given by Eq. (35) is derived using Lyapunov synthesis approach (Ioannou \& Sun, 1996). In addition, in order to ensure parameter convergence, $R_{\eta}(\phi, \theta)$ will need to satisfy the persistence of excitation condition (Ioannou \& Sun, 1996), that is:

$$
\alpha_{1} I \geq \frac{1}{T_{0}} \int_{t}^{t+T_{0}} R_{\eta}(\phi, \theta)^{T} R_{\eta}(\phi, \theta) d \tau \geq \alpha_{0} I
$$

for some constants $\alpha_{1} \geq \alpha_{0}>0, T_{0}>0$, and for all $t \geq T_{\omega}$, where $T_{\omega}$ is the gyroscope fault detection time. Let us define the attitude angle estimation error as $\tilde{\eta} \triangleq \eta-\hat{\eta}$ and the bias estimation error as $\tilde{b}_{\omega} \triangleq \hat{b}_{\omega}-b_{\omega}$. The stability and learning performance of the adaptive estimation scheme Eq. (35) - Eq. (36) is given below.

Theorem 2. Suppose that a gyroscope sensor bias fault occurs at time $t_{\omega} \geq 0$ and is detected at some time $T_{\omega}>t_{\omega}$. Then, if the PE condition given by Eq. (37) is satisfied, the adaptive scheme described by Eq. (35) - Eq. (36) guarantees that $\mathbb{E}(\tilde{\eta})$ and $\mathbb{E}\left(\tilde{b}_{\omega}\right)$ converge to zero exponentially.

Proof. The state and parameter estimation error dynamics are given by

$$
\begin{aligned}
\dot{\tilde{\eta}} & =-\Lambda_{2} \tilde{\eta}+R_{\eta}(\phi, \theta) \tilde{b}_{\omega}-R_{\eta}(\phi, \theta) n_{\omega} \\
\dot{\tilde{b}}_{\omega} & =-\Gamma R_{\eta}(\phi, \theta)^{T} \tilde{\eta} .
\end{aligned}
$$

Because $n_{\omega}$ is bounded (see Assumption 2), it can be easily shown that all signals involved in Eq. (35) - Eq. (36) are bounded by considering $R_{\eta}(\phi, \theta) n_{\omega}$ as a bounded disturbance term. Additionally, by taking the expectation of Eq. (38) and Eq. (39), and by making use of Assumption 2, we obtain

$$
\begin{aligned}
\frac{d \mathbb{E}(\tilde{\eta})}{d t} & =-\Lambda_{2} \mathbb{E}(\tilde{\eta})+R_{\eta}(\phi, \theta) \mathbb{E}\left(\tilde{b}_{\omega}\right) \\
\frac{d \mathbb{E}\left(\tilde{b}_{\omega}\right)}{d t} & =-\Gamma R_{\eta}(\phi, \theta)^{T} \mathbb{E}(\tilde{\eta}) .
\end{aligned}
$$

Defining $\epsilon_{1} \triangleq \mathbb{E}(\tilde{\eta})$ and $\epsilon_{2} \triangleq \mathbb{E}\left(\tilde{b}_{\omega}\right)$, we can rewrite equations Eq. (40) - Eq. (41) into the following compact form:

$$
\left[\begin{array}{c}
\dot{\epsilon}_{1} \\
\dot{\epsilon}_{2}
\end{array}\right]=\left[\begin{array}{cc}
-\Lambda_{2} & R_{\eta}(\phi, \theta) \\
-\Gamma R_{\eta}(\phi, \theta)^{T} & O
\end{array}\right]\left[\begin{array}{l}
\epsilon_{1} \\
\epsilon_{2}
\end{array}\right]
$$

where $O$ is a $3 \times 3$ zero matrix. By following similar reasoning logics as given in the proof of Theorem 5.2.2 and Lemma 5.6.3 in (Ioannou \& Sun, 1996), it can be shown that $\left(\epsilon_{1}, \epsilon_{2}\right)=(0,0)$ is exponentially stable, hence, concluding the proof. 


\section{EXPERIMENTAL RESUltS}

In this section, real-time experimental results using an indoor quadrotor test environment are described to illustrate the effectiveness of the sensor fault diagnosis algorithm.

\subsection{Experimental Setup}

A block diagram of the experimental system setup is shown in Figure 2. During flight tests, quadrotor position and attitude information is obtained from a Vicon motion capture camera system. Position and Euler angle measurements are collected every $10 \mathrm{~ms}$ and relayed from a Vicon dedicated PC via TCP/IP connection to a ground station computer. As in (Macdonald, Leishman, Beard, \& McLain, 2014), position measurements are corrupted with normal noise with standard deviation of $0.25 \mathrm{~m}$. Additionally, position measurements are down sampled to $1 \mathrm{~Hz}$, in order to further simulate real-world applications. The fault diagnosis method is evaluated in realtime during autonomous flight of a quadrotor built in-house with off-the-shelf components. The quadrotor is equipped with the Qbrain embedded control module from Quanser Inc. The control module consists of a HiQ acquisition card providing real-time IMU measurements, and a Gumstix Duo Vero microcontroller running the real-time control software. An IEEE 802.11 connection between the ground station PC and the Gumstix allows for fast and reliable wireless data transmission and on-line parameter tuning. Position and attitude information obtained from the Vicon system along with trajectory commands generated by the ground station are sent to the quadrotor in order to achieve real-time autonomous flight. The control software executes on-board at $500 \mathrm{~Hz}$, and accelerometer and gyroscope measurement are sampled at $200 \mathrm{~Hz}$. During the experimental stage, the quadrotor is commanded to move in a circular trajectory, while maintaining constant orientation and altitude. As previously shown, the fault diagnosis technique employed in this approach is independent of the structure of the controller. Therefore, for brevity of presentation, the discussion on controller design is purposely omitted in this paper.

In order to evaluate the proposed diagnosis method sensor measurements are artificially corrupted by injecting a constant bias into the accelerometer and gyroscope measurements, respectively, while the quadrotor is airborne. Figure 3 shows the fault time profile of the two types of sensor faults under consideration. As can be seen, an accelerometer fault is introduced at time $t=30 \mathrm{~s}$ until $t=50 \mathrm{~s}$. Between time $t=60 \mathrm{~s}$ and time $t=80 \mathrm{~s}$, a gyroscope bias is injected into the sensor measurements. Additionally, in order to evaluate the performance of the FDIE algorithm in the presence of multiple faults, accelerometer and gyroscope faults are simultaneously injected at time $t=100 \mathrm{~s}$. Flight data is processed on-line, and real-time sensor fault diagnostic decision is generated by the diagnostic algorithm. In the following sections, the fault

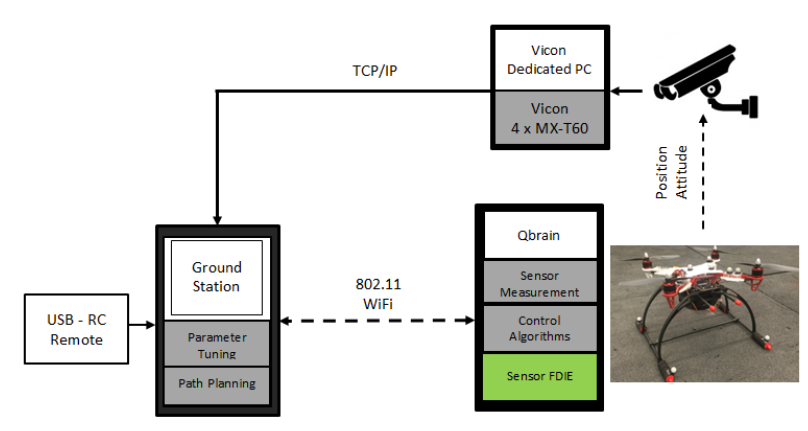

Figure 2. Experimental system architecture setup

diagnosis results are detailed.
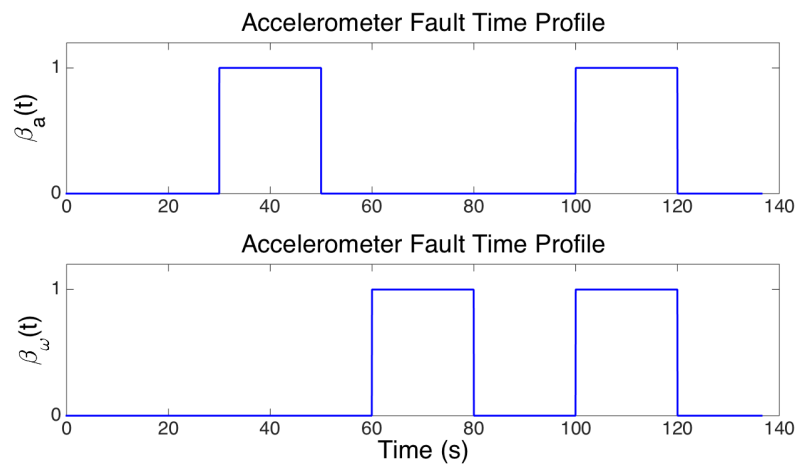

Figure 3. Sensor fault time profile

\subsection{Case of Accelerometer Bias}

The case of an accelerometer measurement bias fault is illustrated in this section. At time $t=30 \mathrm{~s}$, a constant bias $b_{a}=[0.15,0.2,0.75]^{T} \mathrm{~m} / \mathrm{s}^{2}$, is injected into the accelerometer measurements. Figure 4 shows the residuals generated by the two diagnostic estimators described by Eq. (14) and Eq. (19), respectively. In order to enhance the diagnostic decision based on the FDI logic given by Table 1, the two-sided cumulative sum (CUSUM) test is applied to process the diagnostic residuals (Gustafsson, 2000). Figure 5 shows the statistic property generated by the CUSUM test. A fixed threshold is chosen for the detection and isolation of sensor faults. As can be seen, shortly after the occurrence of the fault, at least one component of the test statistic corresponding to the residuals generated by the accelerometer diagnostic estimator exceeds the detection threshold, indicating the occurence of a fault in the accelerometr sensor. On the other hand, all components of the test statistic corresponding to the gyroscope bias remain well below the detection threshold. Based on the detection and isolation logic given in Table 1, it can be concluded that a fault has occurred in the accelerometer measurement. In addition, Figure 6 shows the estimation of the bias in the accelerometer for each axis, respectively. As can be seen, 
the estimate of accelerometer converges closely to the actual value.

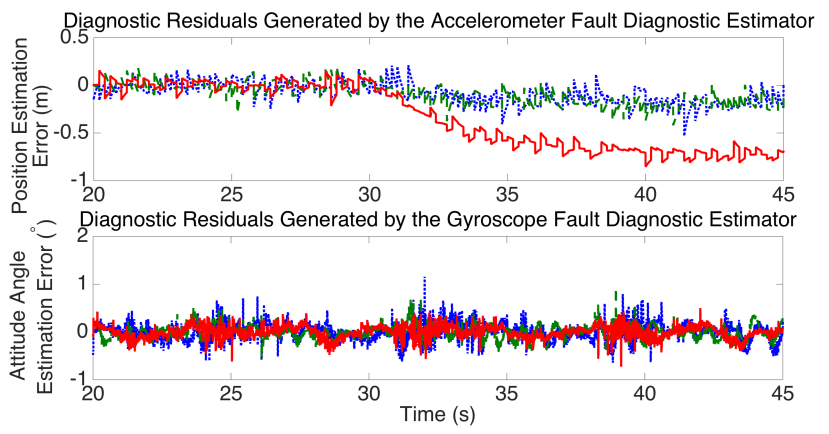

Figure 4. Raw diagnostic residuals: accelerometer bias fault

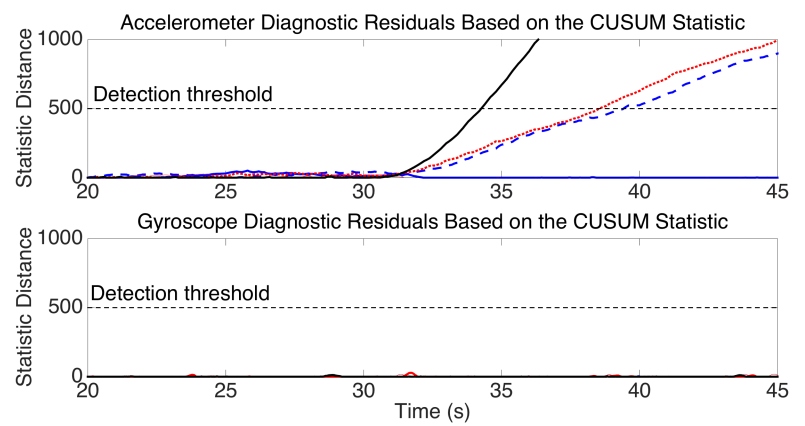

Figure 5. Diagnostic residual generated by CUSUM: accelerometer bias fault.

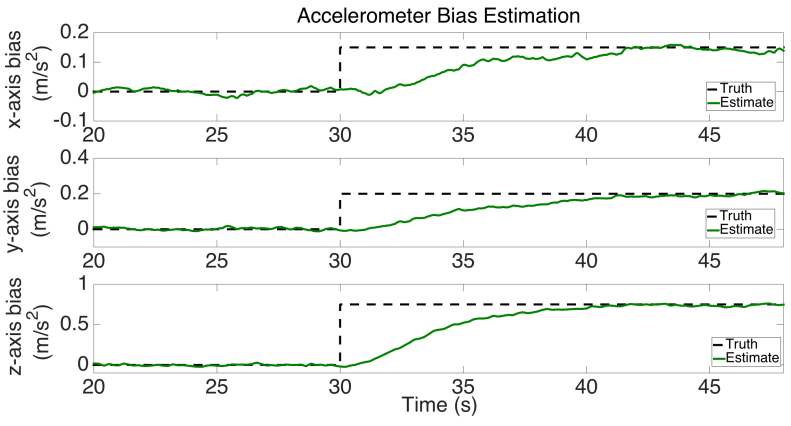

Figure 6. Accelerometer bias estimation.

\subsection{Case of Gyroscope Bias}

A gyroscope bias with $b_{\omega}=[5,-7,-10]^{T} \circ / s$ is injected into the sensor measurements at time $t=60 \mathrm{~s}$. Figure 7 shows the raw diagnostic residuals generated by Eq. (14) and Eq. (19), respectively. The results of the CUSUM test are shown in Figure 8. As can be seen, at least one component of the test statistic corresponding to the gyroscope sensor fault exceeds the detection threshold shortly after fault occurrence. On the other hand, all components of the test statistic corresponding to the accelerometer fault remain well below the detection threshold. Thus, it can be concluded that a fault has occurred in the gyroscope measurement. In addition, Figure 9 shows the estimation of the bias in the gyroscope for each axis, respectively. As can be seen, after a short time, the estimate of gyroscope bias is reasonably close to its actual value.

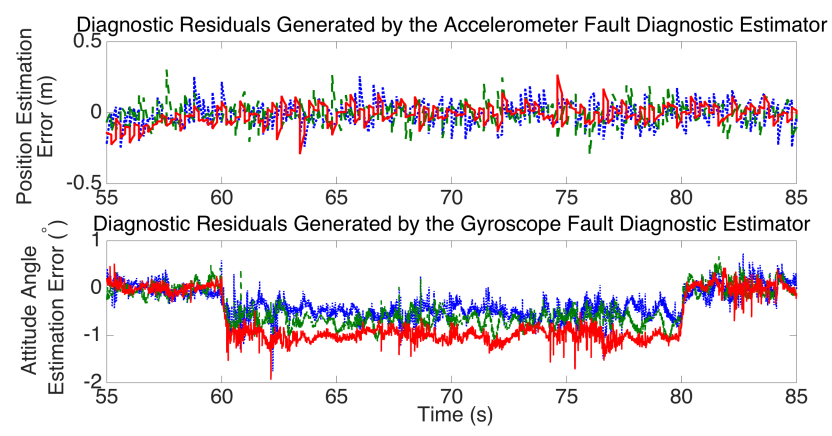

Figure 7. Raw diagnostic residuals: gyroscope bias fault

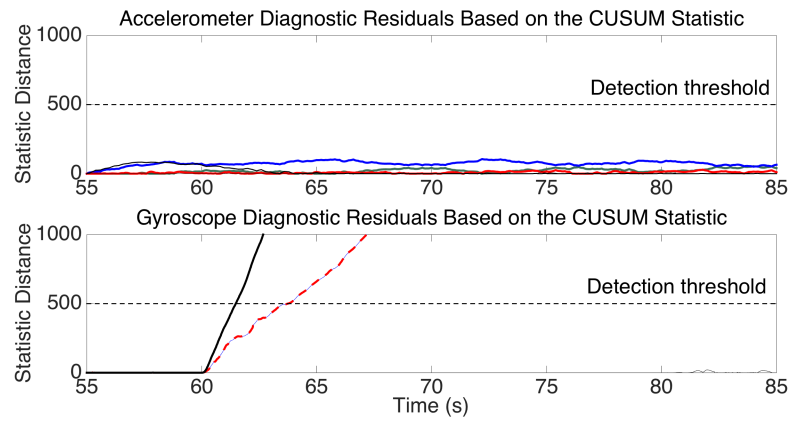

Figure 8. Diagnostic residual generated by CUSUM: gyroscope bias fault.

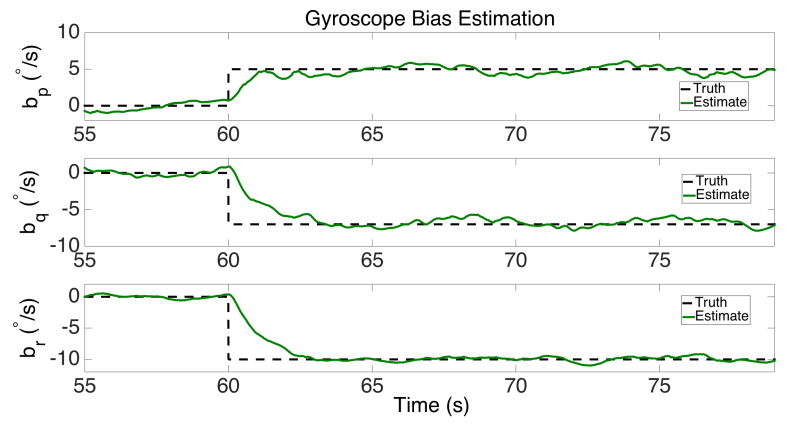

Figure 9. Gyroscope bias estimation.

\subsection{Case of Simultaneous Faults}

The case of simultaneous accelerometer and gyroscope faults is also considered. Specifically, at time $t=100 \mathrm{~s}$, biases $b_{a}=[0.15,0.2,0.75]^{T} \mathrm{~m} / \mathrm{s}^{2}$ and $b_{\omega}=[5,-7,-10]^{T \circ} / \mathrm{s}$ are injected into accelerometer and gyroscope measurements, respectively. Figure 10 shows the statistic property generated by the CUSUM test. As can be seen, shortly after the occur- 


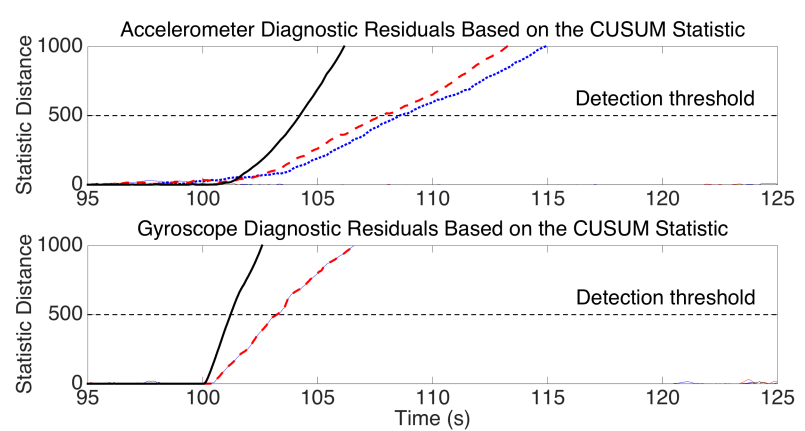

Figure 10. Diagnostic residual generated by CUSUM: simultaneous sensor faults.

rence of the faults, the test statistics corresponding to both diagnostic estimators, exceed their respective detection thresholds. Therefore, it can be concluded that faults have occurred in both accelerometer and gyroscope measurements. Furthermore, Figure 11 and Figure 12 show the estimation of the accelerometer and gyroscope biases, respectively. As can be seen, estimation results are satisfactory.
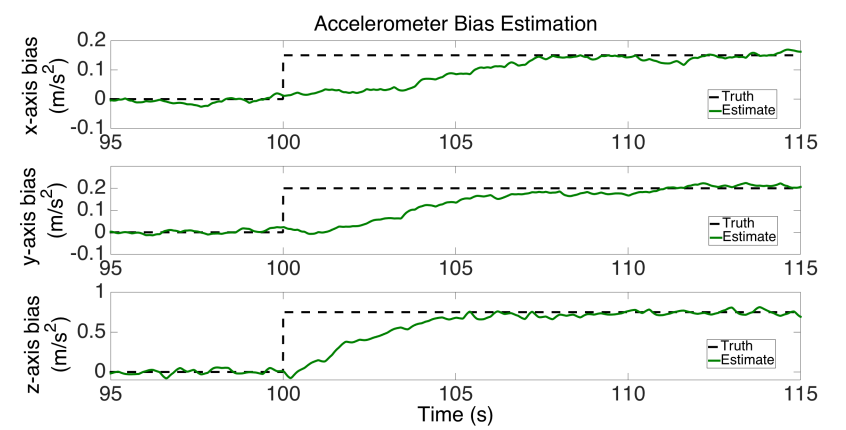

Figure 11. Accelerometer bias estimation in the simultaneous fault occurrence scenario.

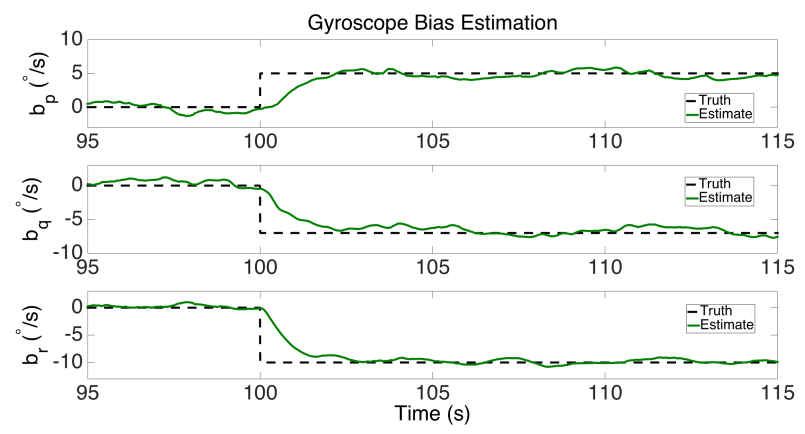

Figure 12. Gyroscope bias estimation in the simultaneous fault occurrence scenario.

\section{Conclusion And Future Work}

In this paper, the design, analysis, and real-time experimental results of a nonlinear fault diagnostic method for sensor bias faults in accelerometer and gyroscope measurements of quadrotor UAVs is presented. Based on the idea that accelerometer and gyroscope measurements coincide with translational forces and rotational and moments acting on the UAV body frame, respectively, two FDI estimators are designed to generate structured residuals for fault detection and isolation. In addition, nonlinear adaptive estimation estimation schemes are developed to provide an estimate of the sensor bias. The proposed diagnostic method is implemented on a quadrotor UAV test environment and demonstrated during real-time autonomous flight. An interesting direction for future research is to develop and demonstrate a systematic fault diagnostic and fault-tolerant control method for quadrotor actuator faults.

\section{REFERENCES}

Anderson, B. D. O., Bitmead, R. R., Johnson, C. R., Jr., Kokotovic, P. V., Kosut, R. L., Mareels, I. M., ... Riedle, B. D. (1986). Stability of Adaptive Systems: Passivity and Averaging Analysis. Cambridge, Massachusettes: The MIT Press.

Bangura, M., \& Mahony, R. (2012). Nonlinear dynamic modelling for high performance control of a quadrotor. In Proceedings of Austrasian Conference on Robotics and Automation.

Bastin, G., \& Gevers, M. (1988). Stable adaptive observers for nonlinear time-varying systems. IEEE Transactions on Automatic Control, 33(7), 650-658.

Castillo, P., Lozano, R., \& Dzul, A. (2005). Modelling and Control of Mini-Flying Machines. Berlin: SpringerVerlag.

Dydek, Z. T., Annaswamy, A. M., \& Lavretsky, E. (2013). Adaptive control of quadrotors UAVs: A design trade study with flight evaluations. IEEE Transaction on Automatic Control Systems Technology, 21(4), 14001406.

Freddi, A., Longhi, S., \& Monteriú, A. (2009). A modelbased fault diagnosis system for a mini-quadrotor. In 7th Workshop on Advanced Control and Diagnosis.

Guenard, N., Hamel, T., \& Mahony, R. (2008). A practical visual servo control for an unmanned aerial vehicle. IEEE Transaction on Robotics, 24(2), 331-340.

Gustafsson, F. (2000). Adaptive filtering and change detection. Wiley, West Sussex, England.

Ioannou, P. A., \& Sun, J. (1996). Robust Adaptive Control. Mineola, New York: Dover Publications, Inc.

Ireland, M., \& Anderson, D. (2012). Development of navigation algorithms for NAP-of-the-earth UAV flight in a constrained urban environment. In 28th International Congress of the Aeronautical Sciences.

Lantos, B., \& Marton, L. (2011). Nonlinear Control of Vehicles and Robots. In (chap. Nonlinear Control of Airplanes and Helicopters). Springer-London.

Leishman, R. C., McDonald, J. C., Beard, R. W., \& McLain, 
T. (2014). Quadrotors and accelerometers. State estimation with an improved dynamic model. IEEE Control Systems Magazine, 34(1), 28-41.

Macdonald, J., Leishman, R., Beard, R., \& McLain, T. (2014). Analysis of an improved IMU-based observer for multirotor helicopters. Journal of Intelligent Robotic Systems, 64, 1049-1061.

Martin, P., \& Salaün, E. (2010). The true role of accelerometer feedback in quadrotor control. In IEEE International Conference on Robotics and Automation.

Pounds, P., Mahony, R., \& Gresham, J. (2004). Towards dynamically-favourable quad-rotor aerial robots. In Australasian Conference on Robotics and Automation, ACRA.

Shima, T., \& Rasmussen, S. (2008). UAV Cooperative Decision and Control: Challenges and Practical Approaches.

US Dept. of Defense. (2012). Unmanned systems integrated roadmap FY2011-2036 (Tech. Rep.). Secretary of Defense, Washington, D.C.

Vachtsevanos, G., Tang, L., Drozeski, G., \& Gutierrez, L. (2005). From mission planning to flight control of unmanned aerial vehicles: Strategies and implementation tools. Anual Reviews in Control, 29, 101-115.

Zhang, X. (2011). Sensor bias fault detection and isolation in a class of nonlinear uncertain systems using adaptive estimation. IEEE Transaction on Automatic Control, 56(5), 1220-1226.

Zhang, X., Polycarpou, M., \& Parsini, T. (2001). Robust fault isolation for a class of non-linear input-output systems. International Journal Control, 74(13), 1295-1310.

Zhang, Y., Chamseddine, A., Rabbath, C., Gordon, B., Su, C., Rakheja, S., ... Gosselin, P. (2013). Development of advanced FDD and FTC techniques with application to an unmanned quadrotor helicopter testbed. Journal of the Franklin Institue, 350(9), 2396-2422.

\section{BIOGRAPHIES}

Remus C. Avram is a PhD candidate at Wright State Univerisity, Dayton OH. Remus, received his B.S. in Electrical Engineering with concetration in Computer Engineering at University of Texas, San Antonio, Texas in 2009. In 2011, Remus obtained his M.S. in Electrical Engineering with concetration in Control Systems from Wright State University, Dayton, Ohio. He is currently pursuing a doctoral degree in Electrical Engineering. Remus's main research include nonlinear health diagnostic methods and real-time intelligent control.

Xiaodong Zhang received the B.S. degree from Huazhong University of Science and Technology, Wuhan, China, the M.S. degree from Shanghai Jiao Tong University, Shanghai, China, and the Ph.D. degree from University of Cincinnati, Cincinnati, OH, USA, all in electrical engineering, in 1994,
1997 and 2001, respectively. He is currently an Associate Professor of Electrical Engineering Department, Wright State University, Dayton, OH. His research interests include intelligent control systems, fault diagnosis and prognosis, faulttolerant control, verification and validation of control systems for safety assurance, etc. He is an Associate Editor of the IEEE Transactions on Control Systems Technology and a member of the IFAC SAFEPROCESS Technical Committee.

Jonathan Muse received an undergraduate degree from the University of Alabama in 2005, a Masters Degree from the Georgia Institute of Technology in 2008, and a PhD in Aerospace Engineering from the Georgia Institute of Technology in 2010. $\mathrm{He}$ is currently a research engineer for the Aerospace Systems Directorate at the Air Force Research Lab (AFRL). At AFRL, he is responsible for executing basic research related to hypersonic vehicle control and advanced methods in nonlinear control. He is the Co-PI for HIFiRE flight 6, an adaptive flight control experiment on a scramjet hypersonic vehicle currently being constructed. He also holds an adjunct professor position at Wright State University in the Electrical Engineering department where he teaches control theory and UAV flight control. In addition, he also serves on various advisory boards for both industry and academia. 